\title{
Continuous self-imaging regime with a double-grating mask
}

\author{
Luis Miguel Sanchez-Brea, ${ }^{*}$ Francisco Jose Torcal-Milla, \\ and Eusebio Bernabeu \\ Optics Department, Applied Optics Complutense Group, Universidad Complutense de Madrid, \\ Facultad de Ciencias Físicas, Ciudad Universitaria s.n., 28040 Madrid, Spain \\ * *Corresponding author: sanchezbrea@ fis.ucm.es
}

Received 14 July 2009; revised 24 September 2009; accepted 1 October 2009; posted 2 October 2009 (Doc. ID 114144); published 13 October 2009

\begin{abstract}
We analyze the Talbot effect produced by a mask composed of two diffraction gratings. Combinations with phase and amplitude gratings have been studied in the near-field regime. For a two-phase-gratings configuration, the Talbot effect is canceled, even when using monochromatic light; that is, the intensity distribution is nearly independent of the distance from the mask to the observation plane. Therefore, the mechanical tolerances of devices that use the Talbot effect may be improved. In addition, the spatial frequency of the fringes is quadrupled, which improves the accuracy of devices that employ this mask. An experimental verification for the best case two phase gratings, has also been performed, validating the theoretical results. (C) 2009 Optical Society of America

OCIS codes: $\quad 050.1950,230.1950,050.2770$.
\end{abstract}

\section{Introduction}

Diffraction gratings are used in several important fields of science, such as chemistry, biology, astrophysics, photonics, mechanical engineering, robotics, and machine tool [1-4]. In particular, gratings are an important part of optical encoders for measuring the displacement between a fixed scale and a moving reading head [5]. The scale is a glass or steel diffraction grating and the reading head is formed by a mask, with several diffraction gratings, photodetectors, and conditioning electronics. The reading head moves along the scale and the diffraction pattern produced by the scale and the mask provides an optical modulation of the illumination field that is transformed to an electrical signal and used to obtain the relative or absolute displacement between them. The correct functioning of these devices depends strongly on the distance between scale and mask, since the Talbot effect is present [1]. When a collimated and monochromatic light beam impinges on

0003-6935/09/305722-06\$15.00/0

(C) 2009 Optical Society of America a diffraction grating, self-images of the grating are obtained only at certain distances, called Talbot planes, [6,7]. The distance between Talbot planes depends on the period of the grating $p$ and the wavelength of the illumination source $\lambda, z_{T}=n p^{2} / \lambda$, where $n$ is an integer. In a general description of the problem, the mask should be placed at a distance equal to an integer number of Talbot distances. When this does not happen, the optical encoder does not work properly since the self-images present a low contrast or even disappear. As a consequence, the mechanical tolerances are restricted by the Talbot effect, which is an important handicap in optical encoders.

Some works about cancellation or pseudocancellation of the Talbot effect with polychromatic light [8] and in double-grating systems [9] have been published. When the Talbot effect is canceled, then the intensity distribution is nearly independent of the distance from the grating to the observation plane. The Talbot effect can also be canceled when special gratings are used $[10,11]$. In this work, we propose the use of a mask composed by two conventional lamellar gratings to cancel the Talbot effect. 
We have theoretically analyzed masks composed of two amplitude gratings, two phase gratings, phaseamplitude gratings, and amplitude-phase gratings. Pseudo-cancellation of the Talbot effect occurs for amplitude-amplitude, amplitude-phase, and phaseamplitude. Under certain conditions, the Talbot effect is totally canceled for the case of a phase-phase mask, In addition, for this phase-phase configuration, the spatial frequency of the self-images is 4 times higher than that of the grating. This fact allows improving the accuracy in devices that employ this mask. We also have performed an analysis of the tolerances in the positioning of the gratings in the mask in order to verify the reliability in the fabrication process. Finally, an experimental corroboration for the most favorable case, the phase-phase mask, is carried out. The experimental results validate the proposed double-grating mask for obtaining continuous self-imaging.

\section{Theoretical Approach}

Let us consider a monochromatic plane wave with wavelength $\lambda$ that illuminates a mask formed by two gratings with the same period, $p$, and separated by a distance $z_{1}$ (Fig. 1). The intensity just after the second grating corresponds with the classical description of moiré given by Patorsky [12]:

$$
\begin{aligned}
I\left(x, z_{1}\right)= & \sum_{n, n^{\prime}, m, m^{\prime}} a_{n} a_{n^{\prime}}^{*} b_{m} b_{m^{\prime}}^{*} \exp \left\{i q x \left[\left(n-n^{\prime}\right)\right.\right. \\
& \left.\left.+\left(m-m^{\prime}\right)\right]\right\} \exp \left[i \frac{q^{2}}{2 k}\left(n^{2}-n^{\prime 2}\right) z_{1}\right],
\end{aligned}
$$

where $q=2 \pi / p$ and $a_{n}$ and $b_{m}$ are the coefficients of the Fourier series expansion of the diffraction grat-

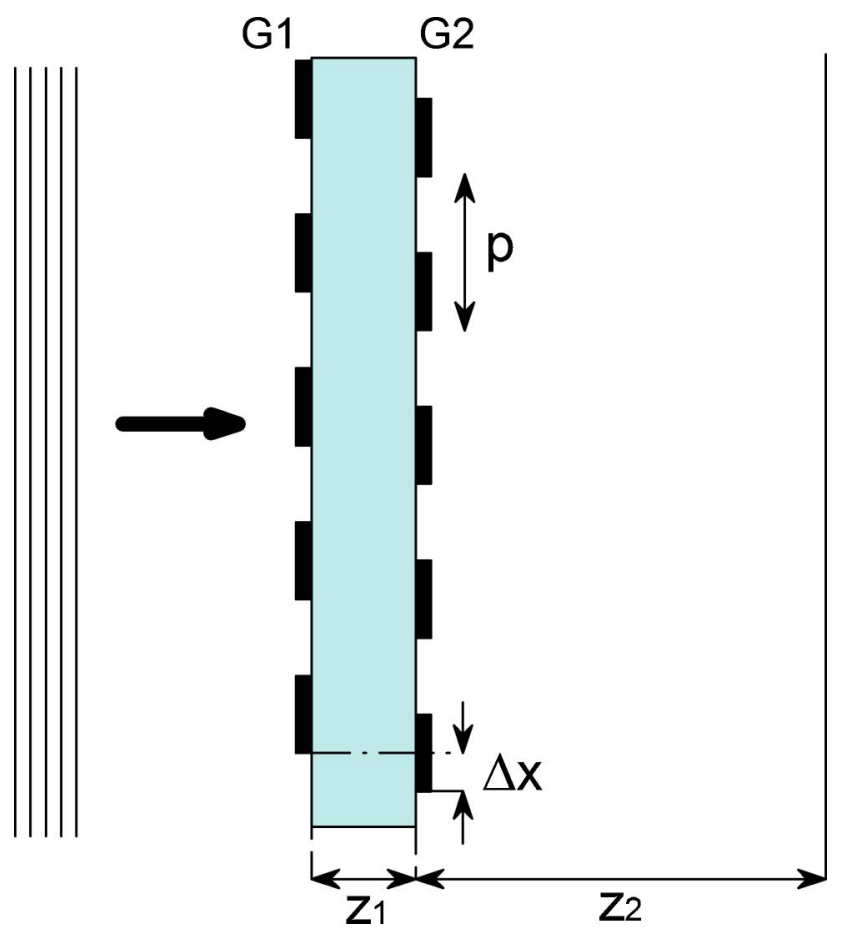

Fig. 1. (Color online) General scheme of the mask. ings, respectively. On the other hand, considering that the observation plane is placed at a distance $z_{2}$ after the second grating, the more general expression results in [13]

$$
\begin{aligned}
& I\left(x, z_{1}, z_{2}\right)=\sum_{n, n^{\prime}, m, m^{\prime}} a_{n} a_{n^{\prime}}^{*} b_{m} b_{m^{\prime}}^{*} \exp \left\{i q x \left[\left(n-n^{\prime}\right)\right.\right. \\
& \left.\left.\quad+\left(m-m^{\prime}\right)\right]\right\} \exp \left[i \frac{q^{2}}{2 k}\left(n^{2}-n^{\prime 2}\right)\left(z_{1}+z_{2}\right)\right] \\
& \quad \times \exp \left[i \frac{q^{2}}{2 k}\left(m^{2}-m^{\prime 2}\right) z_{2}\right] \exp \left[i \frac{q^{2}}{k}\left(n m-n^{\prime} m^{\prime}\right) z_{2}\right] .
\end{aligned}
$$

Including a relative displacement $\Delta x$ between the gratings along the $x$ axis and performing the following changes of variable, $N=n-n^{\prime}, M=m-m^{\prime}$, $u=n-N / 2$, and $v=m-M / 2$, Eq. (2) results in

$$
\begin{aligned}
& I\left(x, z_{1}, z_{2}\right)=\sum_{N, M, u, v} a_{u+N / 2} a_{u-N / 2}^{*} b_{v+M / 2} b_{v-M / 2}^{*} \\
& \quad \times \exp [i q x(N+M)] \exp (-i M q \Delta x) \times \exp \left(2 \pi i u N \frac{z_{1}}{z_{T}}\right) \\
& \quad \times \exp \left[i 2 \pi(N+M)(u+v) \frac{z_{2}}{z_{T}}\right],
\end{aligned}
$$

where $z_{T}$ is the Talbot distance when the medium between the gratings is not a vacuum but is a dielectric material with refraction index $\tilde{n}$.

In Subsections 2.A-2.C, we will consider the four possible combinations among amplitude and phase gratings for the mask design. We will take advantage of the fact that, for an amplitude grating, the Fourier coefficients fulfill the relationships $a_{n}=a_{n}^{*}=a_{-n}$, and, for a lamellar phase grating, $b_{n}=b_{-n}$.

To obtain a continuous self-imaging regime, we will also consider that the phase retardation between both levels in phase gratings corresponds to $\pi / 2$, the distance between the gratings is $z_{1}=z_{T} / 2$, and the relative displacement between them is $\Delta x=p / 4$. The choice of these parameters is a consequence of numerical simulations, checking all the possible values that are $0<z_{1} \leq z_{T}$ and $0 \leq \Delta x<p$. In addition, the phase retardation for phase gratings is chosen in order to obtain a total maximum delay of $\pi$ considering two phase gratings, First, we will consider $0, \pm 1$ diffraction orders so that simple analytical solutions can be obtained. The values for the Fourier coefficients are $a_{0}=1 / 2$ and $a_{1}=a_{-1}=1 / \pi$ for the amplitude grating, and $b_{0}=-(1+i) / 2$ and $b_{1}=b_{-1}=$ $(1-i) / \pi$ for the phase grating. For amplitude gratings, the analysis with orders $0, \pm 1$ is quite simple and provides a crude estimation of their behavior, useful enough for our purposes. On the other hand, for the phase gratings used in this work, $90 \%$ of the total intensity is at these three diffraction orders, and it provides a good approximation. In addition, in Section 3, we compare the results obtained with this approximation to the numerical results for a much higher number of terms. 


\section{A. Amplitude-Phase and Phase-Amplitude Mask}

When the first grating is an amplitude grating and the second grating is a phase grating, and considering only orders $0, \pm 1$, Eq. (3) results in

$$
\begin{aligned}
& I_{\mathrm{AP}}\left(x, Z_{2}\right)=a_{0}^{2}\left(b_{0}^{2}+2 b_{1}^{2}\right)+2 a_{1}^{2}\left(b_{0}^{2}+b_{1}^{2}\right) \\
& \quad-4 a_{0} a_{1} \cos (q x)\left[b_{0}^{2} \sin \left(\pi Z_{2}\right)+b_{1}^{2} \sin \left(3 \pi Z_{2}\right)\right] \\
& \quad+4 \sin (q x) \operatorname{Re}\left(b_{1} b_{0}^{*}\right)\left[a_{0}^{2} \cos \left(\pi Z_{2}\right)+a_{1}^{2} \cos \left(3 \pi Z_{2}\right)\right] \\
& \quad+2\left(a_{1}^{2} b_{0}^{2}-a_{0}^{2} b_{1}^{2}\right) \cos (2 q x) \\
& \quad-4 I m\left(b_{1} b_{0}^{*}\right) \sin (2 q x) a_{0} a_{1}\left[1-\sin \left(4 \pi Z_{2}\right)\right] \\
& \quad+4 a_{0} a_{1} b_{1}^{2} \cos (3 q x) \sin \left(3 \pi Z_{2}\right) \\
& \quad+4 a_{1}^{2} \operatorname{Re}\left(b_{1} b_{0}^{*}\right) \sin (3 q x) \cos \left(3 \pi Z_{2}\right) \\
& \quad-2 a_{1}^{2} b_{1}^{2} \cos (4 q x)
\end{aligned}
$$

where $Z_{2}=z_{2} / z_{T}$. This equation depends on the distance to the observation plane $z_{2}$ and, in addition, it does not provide us much information. Nevertheless, when we particularize to two Ronchi gratings, the intensity pattern after the second grating simplifies to

$$
\begin{aligned}
& I_{\mathrm{AP}}\left(x, Z_{2}\right)=\frac{8}{\pi^{4}}\left[\left(1+\pi^{2}\right)-\pi^{2} \cos (2 q x)\right. \\
& \left.\quad-2 \pi \cos (q x) \sin ^{2}(q x) \sin \left(3 \pi Z_{2}\right)-\cos (4 q x)\right] .
\end{aligned}
$$

In Eq. (5), the second and fourth terms do not produce contrast modulation along the $z$ axis, but they correspond to harmonic signals. The second term produces constant fringes, but with double frequency. On the other hand, the fourth term also produces constant fringes, but with quadrupled frequency. The first term is a constant level and the third term produces a kind of Talbot effect, since it has periodicity along the $x$ and $z$ axes.

As is shown in Fig. 2(a), although the Talbot effect is not totally cancelled, there is no contrast inversion and a pseudo-cancellation of the Talbot effect is



(b)

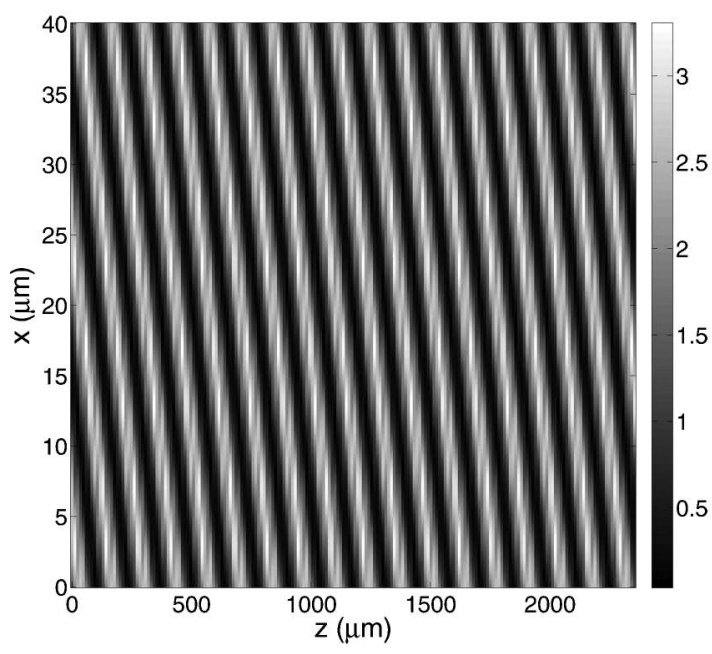

(c)

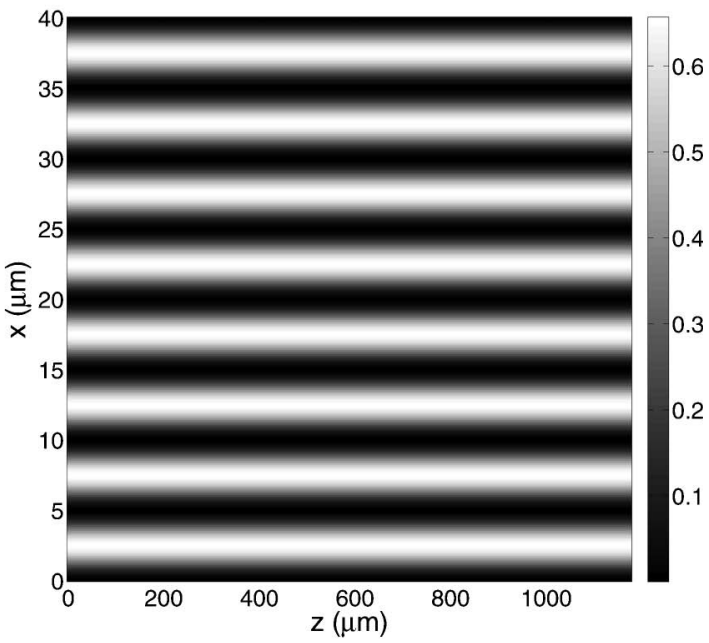

(d)

Fig. 2. Intensity distribution produced by the double-grating mask considering only 0 and \pm 1 orders: (a) amplitude-phase mask, (b) phase-amplitude mask, (c) amplitude-amplitude mask, and (d) phase-phase mask. 
achieved. Bright and dark fringes are curly, but they are acceptable in the usual applications since these short-range variations are normally filtered by photodetectors.

In a similar approach, when the first grating is a phase grating and the second is an amplitude grating, the situation is very similar to the previous case. The intensity pattern in this case results

$$
\begin{aligned}
& I_{\mathrm{PA}}\left(x, Z_{2}\right)=\frac{8}{\pi^{4}}\left[\left(1+\pi^{2}\right)+\pi^{2} \cos (2 q x)\right. \\
& \left.\quad+4 \pi \cos (q x) \cos (2 q x) \cos \left(3 \pi Z_{2}\right)-\cos (4 q x)\right] .
\end{aligned}
$$

The intensity pattern produced by this mask is shown in Fig. 2(b), and it is equivalent to that of the amplitude-phase mask but with a shift of $p / 4$ along the $x$ axis in the fringe pattern.

In both cases, the frequency of the fringes is doubled, which improves the accuracy of the devices that use this kind of mask.

\section{B. Amplitude-Amplitude Mask}

Now, we consider that both gratings are amplitude gratings. We can apply the following relationships to the Fourier coefficients of both gratings: $a_{n}=$ $a_{n}^{*}=a_{-n}$ and $b_{n}=b_{n}^{*}=b_{-n}$. Thus, taking into account the same diffraction orders as in the previous cases, the intensity results in

$$
\begin{aligned}
I_{\mathrm{AA}}\left(x, Z_{2}\right)= & \frac{1}{\pi^{4}}\left\{\left(2+4 \pi^{2}+\pi^{4}\right)-2 \cos (4 q x)\right. \\
& +4 \pi \sin (2 q x)\left[2 \cos \left(q x+3 \pi Z_{2}\right)\right. \\
& \left.\left.-\pi \sin \left(4 \pi Z_{2}\right)\right]+4 \pi^{3} \sin \left(q x-\pi Z_{2}\right)\right\} .
\end{aligned}
$$

The intensity distribution after the second grating is shown in Fig. 2(c). The Talbot effect almost disappears, but the fringes are not perpendicular to the grating.

\section{Phase-Phase Mask}

Finally, let us consider a mask formed by two phase lamellar gratings. Then, the Fourier coefficients of the gratings present the following properties: $a_{n}=a_{-n}$ and $b_{n}=b_{-n}$. As a consequence, the intensity simplifies to

$$
I_{\mathrm{PP}}\left(x, Z_{2}\right)=\frac{32}{\pi^{4}}[1-\cos (4 q x)]=\frac{64}{\pi^{4}} \sin ^{2}(2 q x) .
$$

In this case, the dependence of the intensity pattern in terms of $Z_{2}$ disappears. That is, the Talbot effect is canceled. In addition, the frequency of the fringes quadruples, which allows an increase in the accuracy of the devices that use this configuration. Also, this equation is completely independent of $z_{1}$, which is quite interesting for the manufacturability of the proposed double-grating mask. In Fig. 2(d), the intensity distribution is shown for the case of considering only orders 0 and \pm 1 . In Section $\underline{3}$, a more



Fig. 3. Intensity distribution produced by the phase-phase mask when $-11, \ldots, 11$ orders have been considered. The period of the gratings is $p=20 \mu \mathrm{m}$. A slight dependence with the distance between the mask and the observation plane is observed, but continuous self-imaging is still obtained.

detailed analysis of this phase-phase configuration for the mask is performed.

\section{Detailed Analysis of Phase-Phase Mask}

The result obtained in Eq. (8) only considers the diffraction orders 0 and \pm 1 because the equations are simpler and it gives us an approximation of the intensity distribution since most of the total intensity is directed to these three diffraction orders. Nevertheless, all diffraction orders involved should be considered. In Fig. 3, the intensity distribution of Eq. (3) of the fringes is shown when $-11,-9, \ldots, 9,11$ orders are considered. This means that nearly $100 \%$ of the total intensity is considered. We see that the presented structure is still valid for a continuous selfimaging regime.

An error that can be produced in the system is the relative displacement of the gratings along the $z$ axis. This error is not important because Eq. (8) does not depend on the distance $z_{1}$ between the gratings. Then, small mistakes in the $z_{1}$ location do not affect the diffraction pattern. When an error in the location $z_{1}$ is produced, the intensity distribution according to Eq. (3) is very similar to the ideal situation given in Fig. $\underline{3}$.

\section{Experimental Results}

As is shown in Section 3, the phase-phase mask is the best configuration to obtain continuous selfimaging. Now, we will analyze the experimental behavior of this mask. The setup is shown in Fig. 4 . We used a collimated laser diode (Monocrom, $\lambda=$ $650 \mathrm{~nm}$ ) as and illumination source and, as a photodetector, a CMOS camera ( $\mu$ eye, Imaging Development Systems; pixel size $6 \mu \mathrm{m} \times 6 \mu \mathrm{m})$ with a microscope objective. We manufactured two phase 


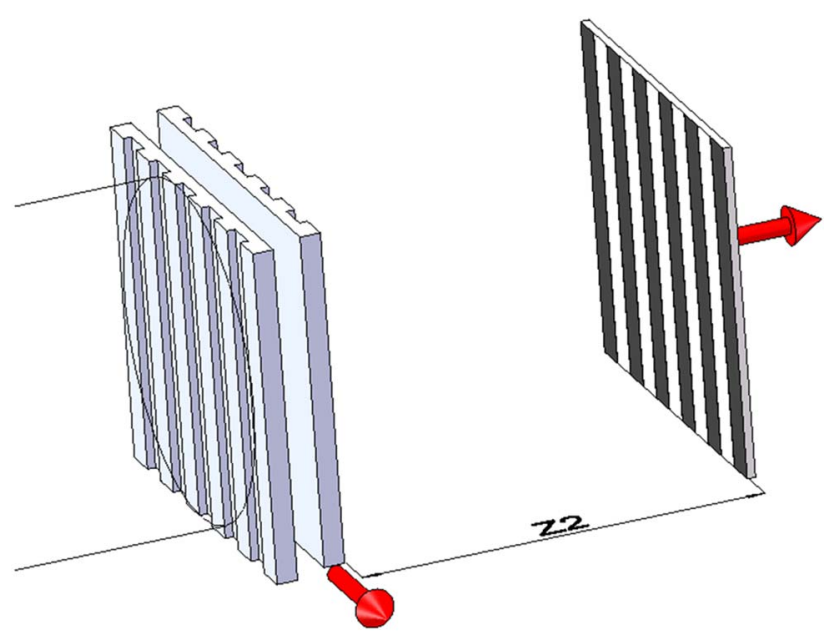

Fig. 4. (Color online) Scheme of the experimental setup.
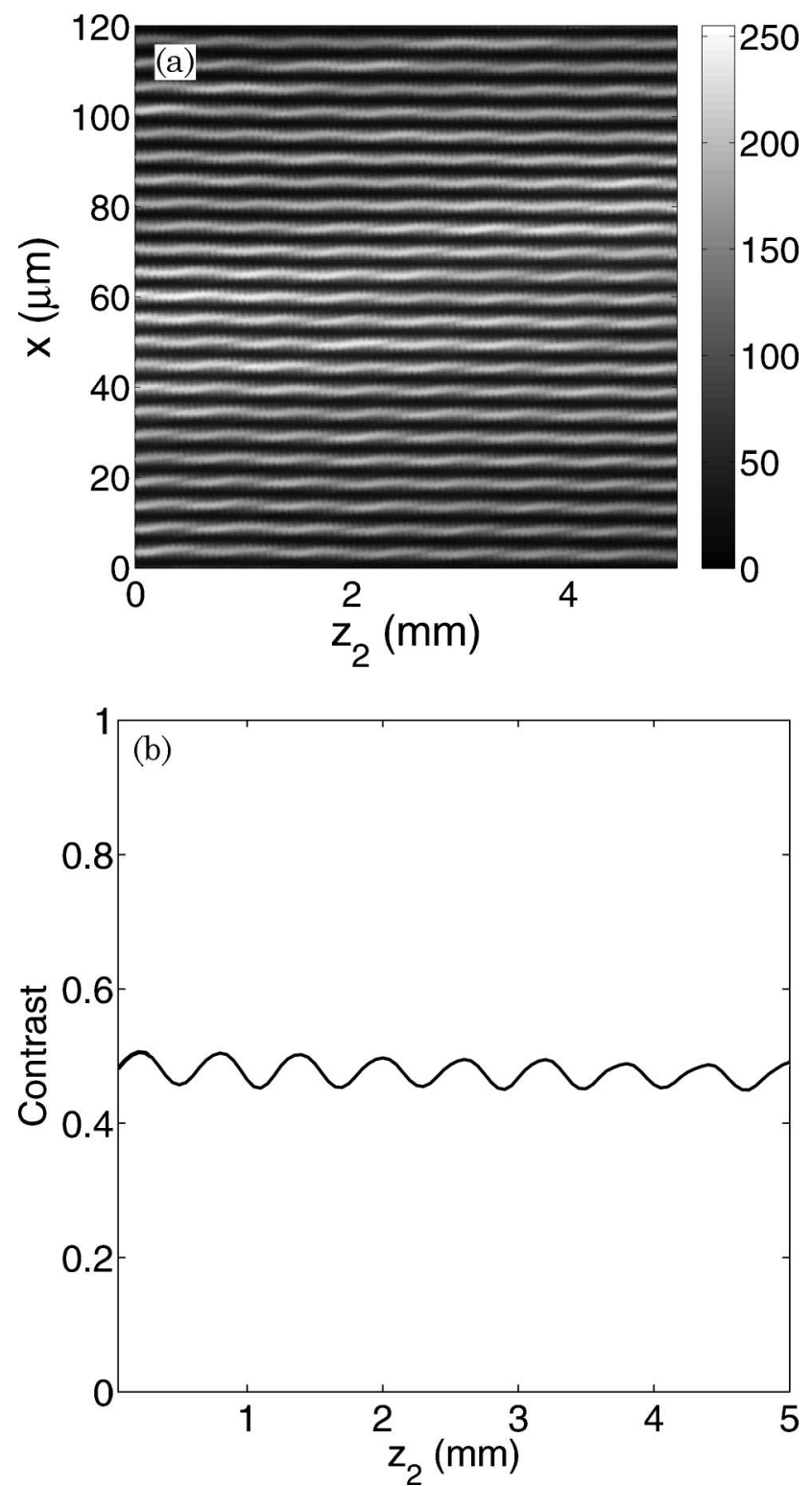

Fig. 5. (a) Experimental intensity distribution of the fringes in terms of the distance $z$ between the mask and the observation plane. (b) Experimental contrast along the $z$ axis. gratings with a period of $p=20 \mu \mathrm{m}$ and a phase retardation of $\pi / 2$ between the levels. For this, we used chrome-on-glass gratings and we applied to them glass etching with fluorhidric acid and, later, chrome etching. The height of the manufactured gratings is around $320 \mathrm{~nm}$ (measured with a confocal microscope), which is quite close to the theoretical phase shift.

The gratings are placed initially one in front of the other at a certain distance. The experimental method consists of separating the camera from the mask along the $z$ axis and acquiring images of the fringes.
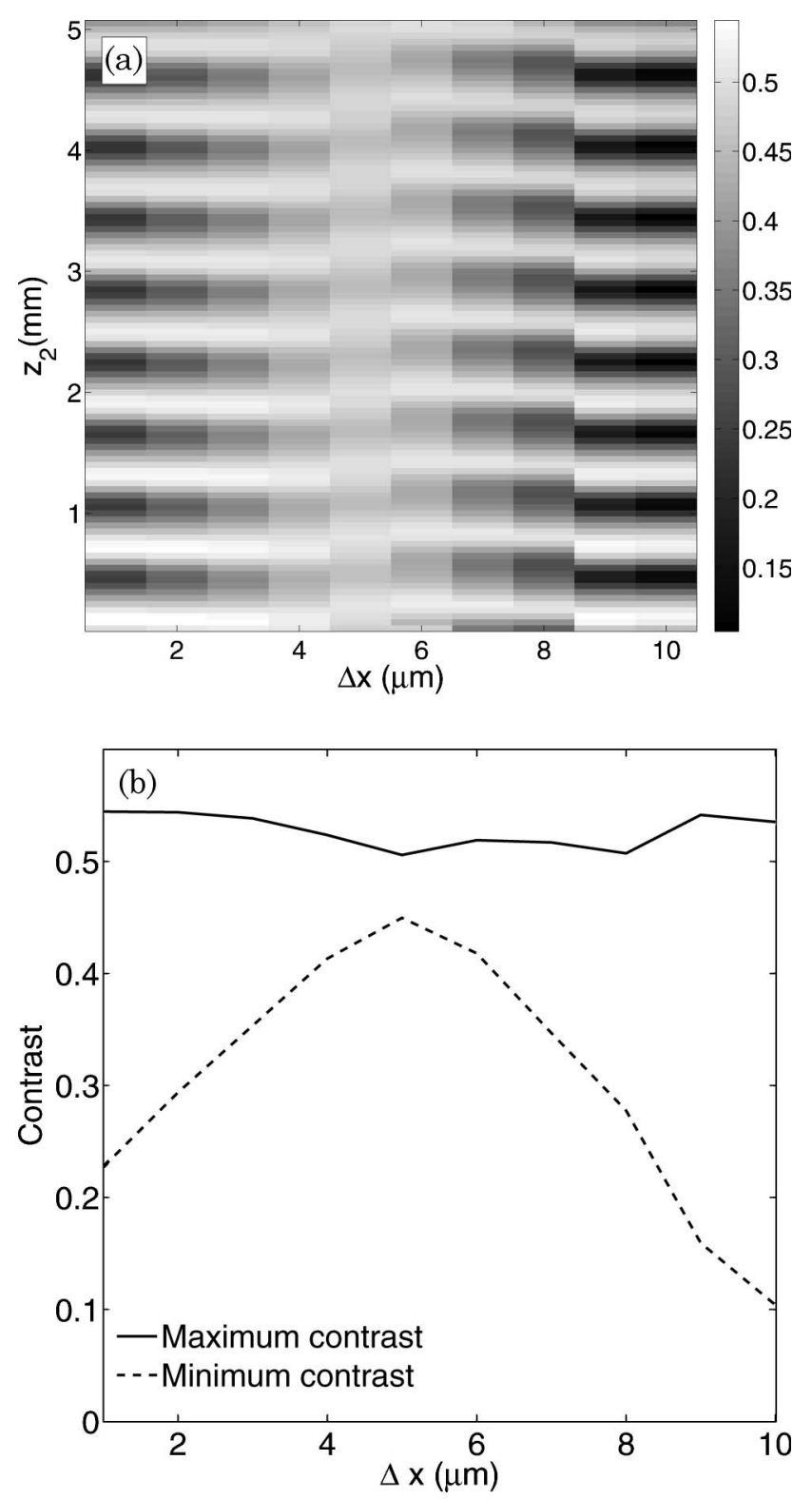

Fig. 6. (a) Experimental contrast of the fringes for the doublegrating mask with the phase-phase configuration in terms of the relative displacement $\Delta x$ between gratings for different values of the distance between the mask and the observation plane. (b) Maximum and minimum experimental contrast for the different relative displacement $\Delta x$ between gratings. 
We have placed the gratings at a relative distance $\Delta x=p / 4$. The experimental results for the Talbot effect cancellation position are shown in Fig. 5(a), which are in agreement with the theoretical prediction. The contrast for the Talbot effect cancellation position for different values of $z_{2}$ is shown in Fig. 5(b). In addition, the contrast of the fringes with respect to the relative position $\Delta x$ between the gratings is shown in Fig. 6(a). At this location, the contrast is quite independent of $z_{1}$; this also happens for variations of $\Delta x= \pm 1 \mu \mathrm{m}$, which allows us manufacturing this device more conveniently.

This tolerance can be observed more clearly in Fig. 6(b), where the maximum and minimum contrasts have been plotted in terms of $\Delta x$. The theoretical results predict contrast equal to 1 for this configuration (phase-phase mask) considering only 0 and \pm 1 diffraction orders, but it is not experimentally obtained, probably because all diffraction orders influence the experimental results.

\section{Conclusions}

In this work, we have shown that a continuous selfimaging regime can be produced using a mask formed by two diffraction gratings. The most successful scheme uses two phase gratings with certain conditions about their phase retardation and their relative position. Then, fringes that depend only slightly on the distance between the mask and the observation plane are formed. In addition, we have experimentally demonstrated the validity of the proposed technique. We have also shown that the tolerance in the positioning between the gratings is great enough to allow for simple fabrication of the doublegrating mask. This kind of mask can be used in optical encoders to improve mechanical tolerances of the devices. In addition, with this mask the fringes quadruple. Therefore, the performance of the systems that use this mask can be improved.

This work has been supported by project CCG08UCM/DPI-3952 of Dirección General de Universidades e Investigación de la Consejería de Educación de la Comunidad de Madrid y Universidad Complu- tense de Madrid and Consorcios Estratégicos Nacionales de Investigación Tecnología project "Tecnologías avanzadas para los equipos y procesos de fabricación de 2015. e-eficiente, e-cológica, e-máquina (eEe)" of the Ministerio de Industria, Turismo y Comercio.

\section{References}

1. E. Keren and O. Kafri, "Diffraction effects in moiré deflectometry," J. Opt. Soc. Am. A 2 (2), 111-120 (1985).

2. A. W. Lohmann and D. E. Silva, "An interferometer based on the Talbot effect," Opt. Commun. 2, 413-415 (1971).

3. B. F. Oreb and R. G. Dorsch "Profilometry by phase-shifted Talbot images," Appl. Opt. 33, 7955-7962 (1994).

4. S. Wei, S. Wu, I. Kao, and F. P. Chiang "Measurement of wafer surface using shadow moire technique with Talbot effect," J. Electron. Packag. 120 166-170 (1998).

5. G. Schirripa Spagnolo, D. Ambrosini, and D. Paoletti, "Displacement measurement using the Talbot effect with a Ronchi grating," J. Opt. A Pure Appl. Opt. 4, S376-S380 (2002).

6. W. H. F. Talbot, "Facts relating to optical science," Philos. Mag. 9, 401-407 (1836).

7. K. Patorski, "The self-imaging phenomenon and its applications," in Progress in Optics, E. Wolf, ed. (North-Holland, 1989), Vol. 27, pp. 1-108.

8. N. Guérineau, B. Harchaoui, and J. Primot, "Talbot experiment re-examined: demonstration of an achromatic and continuous self-imaging regime," Opt. Commun. 180, 199-203 (2000).

9. L. M.Sanchez-Brea, J. Saez-Landete, J. Alonso, and E. Bernabeu "Invariant grating pseudo-imaging using polychromatic light and finite extension source," Appl. Opt. 47, 1470-1477 (2008).

10. L. M. Sanchez-Brea, J. Alonso, and E. Bernabeu "Quasicontinuous pseudoimages for sinusoidal grating imaging using an extended light source," Opt. Commun. 236 53-58 (2004).

11. G. Vincent, R. Haidar, S. Collin, N. Guérineau, J. Primot, E. Cambril, and J. L. Pelouard "Realization of sinusoidal transmittance with subwavelength metallic structures," J. Opt. Soc. Am. B 25, 834-840 (2008).

12. K. Patorsky, Handbook of the Moiré Fringe Technique (Elsevier, 1993).

13. D. Crespo, J. Alonso, and E. Bernabeu, "Generalized grating imaging using an extended monochromatic light source," J. Opt. Soc. Am. A 17, 1231-1240 (2000). 